\title{
High-performance sonographical multimodal imaging of non cystic thyroid lesions: Chances of the preoperative diagnostics in relation to histopathology
}

\author{
M. Brandenstein ${ }^{\mathrm{a}}$, I. Wiesinger ${ }^{\mathrm{a}}$, F. Jung ${ }^{\mathrm{b}}$, C. Stroszczynski ${ }^{\mathrm{a}}$ and E.M. Jung ${ }^{\mathrm{a}, *}$ \\ ${ }^{a}$ Institute of Diagnostic Radiology and Interdisciplinary Ultrasound Department, University Hospital, \\ Regensburg, Germany \\ ${ }^{\mathrm{b}}$ Institute of Biotechnology, Molecular Cell Biology, Brandenburg University of Technology, \\ Senftenberg, Germany
}

\begin{abstract}
.
AIM: To improve preoperative diagnostics of solid non-cystic thyroid lesions by using new high-performance ultrasound techniques: optimized B-mode morphology, elastography, Color-Coded Doppler-Sonography (CCDS) and contrast enhanced ultrasound (CEUS)

MATERIALS AND METHODS: In 33 cases solid, non-cystic thyroid lesions were rated as TIRADS 3 and up from conventional B-mode examinations. Additional high resolution Power Doppler including HR- and Glazing-Flow as optimized macrovascularization techniques, shear wave elastography and CEUS were performed on these patients by one experienced examiner. For CEUS a bolus of 1-2.4 ml Sulfurhexafluorid microbubbles (SonoVue ${ }^{\circledR}$, Bracco, Milan, Italy) was injected into a cubital vein and then the distribution kinetics of the contrast agent were documented from the early arterial phase (10 to 15 seconds after injection) to the late venous phase ( 5 minutes after injection). Postoperative histopathology was the diagnostic gold standard as it provides the most reliable proof.

RESULTS: 33 patients (13 males, 20 females; age 29 - 77 years; mean 55 years; SD 13 years) were included in this study. 28 of them had benign regressive thyroid nodules, 3 had adenomas and 4 were diagnosed with carcinomas ( 3 were histologically identified as papillary thyroid carcinomas, one as a medullary thyroid carcinoma). The volume of the thyroid gland ranged from 6.6 to $401.3 \mathrm{~cm}^{2}$ (mean $72.6 \pm 92.0 \mathrm{~cm}^{2}$ ).

The adenoma diameters ranged from 9 to $40 \mathrm{~mm}$ (mean $22 \pm 16 \mathrm{~mm}$ ) and the carcinoma diameters ranged from 19 to $33 \mathrm{~mm}$ (mean $26 \pm 6 \mathrm{~mm}$ ). The 3 adenomas had different echogenicities: One was completely echofree, one was hypoechoic and one isoechoic. The 4 carcinomas however were equally characterized as hypoechoic and echofree. Two of three adenomas and all of the carcinomas showed an incomplete or diffuse margin. Micro-calcifications were found in one adenoma and in every carcinoma. However, no micro-calcifications were observed in cases of benign regressive nodules.

Performing shear-wave elastography the adenomas showed lower values than the carcinomas: The tissue velocity of the adenomas ranged from $2.86 \mathrm{~m} / \mathrm{s}$ to $3.85 \mathrm{~m} / \mathrm{s}$ (mean $3.32 \pm 0.5 \mathrm{~m} / \mathrm{s}$ ) and in carcinomas from $3.89 \mathrm{~m} / \mathrm{s}$ to $5.66 \mathrm{~m} / \mathrm{s}$ (mean $4.18 \pm 0.3 \mathrm{~m} / \mathrm{s})$.

Marginal hypervascularization was detected in two adenomas after applying CCDS. One adenoma was hypovascularized. The four carcinomas showed an irregular extreme hypervascularization along their margins as well as an irregular central normo- or hypervascularization in CCDS. The additional HR-Flow helped reducing artefacts.

In CEUS the dynamic capillary microvascularization of all carcinomas was very irregular with early enhancement and followed by partial or complete wash-out. In CEUS two adenomas had no wash-out and the other one showed a partial wash-out.
\end{abstract}

\footnotetext{
*Corresponding author: Prof. Dr. Ernst Michael Jung, Institute of Diagnostic Radiology, and Interdisciplinary Ultrasound Department, University Hospital, Regensburg, Germany. Franz-Josef-Strauss Allee 11, 93053 Regensburg, Germany. Tel.: +49 0941944 7409; Fax.: +49 0941944 7409; E-mail: ernst-michael.jung@ukr.de.
} 
CONCLUSION: Using modern multimodal imaging offers new possibilities for the differentiation between benign and malignant thyroid lesions. It is a very important diagnostic tool in addition to the B-Mode TIRADS classification and eases the decision between TIRADS 3, 4 and 5. However, additional multicenter studies are required for more detailed evaluations.

Keywords: Solid thyroid lesions, high-resolution ultrasound, HR-flow, elastography, CEUS, histopathology

\section{Introduction}

Ultrasound is globally the best available imaging method for clarification of possible thyroid pathologies. With increasing age, more and more people tend to develop struma nodosa. Most lesions are normal or complex cysts. B-mode sonography already offers important information about the morphology, size and location of thyroid lesions for later evaluations [1]. With the Color-Coded Doppler-Sonography (CCDS) macrovascularization can be detected. The aim of this study was to combine the most important information in order to evaluate the benignity or malignancy of thyroid lesions and describe it according to the TIRADS classification [3,21].

Trying to assess the sonomorphology of smaller nodules, complicated cystic lesions or multifocal lesions can be difficult. More and more frequently, contrast-enhanced ultrasound (CEUS) is used to capture dynamic macro- and microvascularization of a lesion that showed suspicious findings in B-mode $[2,8,19,22]$. The pattern of enhancement, wash-in after bolus injection of the contrast agent, and the later pattern of disappearance of the enhancement, wash-out, are important CEUS indicators for the differentiation between malignancy and benignity [9]. The tissue stiffness can be evaluated by using elastography. In particular, the shear-wave elastography helps quantifying tissue consolidations [23, 24]. New high-resolution vascular imaging in late contrast phase can be applied for the detection of hemodynamic alterations. In order to take advantage of all these ultrasound techniques, new multifrequency-matrix-linear-probes are utilized. These probes can produce digital Cine-loops, that allow for special post-processing.

The aim of these examinations was to figure out how multimodal ultrasound diagnostics consisting of B-mode, elastography, high-resolution Doppler, CEUS and contrast enhanced special high-resolution vascularization ultrasound can simplify the preoperative characterization of thyroid lesions.

\section{Materials and methods}

The study was approved by the local ethics committee of the University Clinic Regensburg (20-2122104). Every patient signed a declaration of consent before starting CEUS. An experienced sonographer performed every examination (more than 20 years of ultrasound experience with more than 3000 performed examinations per year). Afterwards the reading of the anonymously digitally stored data was performed by two radiologists in consensus.

\subsection{B-mode, CCDS and elastography examination technique}

33 patients with suspect thyroid lesions were examined. Every ultrasound was performed on a high-performance ultrasound device (Resona 7, Mindray) with a multifrequency-matrix-linear-probe (Mindray L9-3U Linear Array transducer, 2.5 to $9.0 \mathrm{MHz}$ ). The examinations were documented with single pictures or Cine-Loops saved as digital DICOM files in PACS. After measuring the size of every single thyroid lobe and the isthmus in three planes, the volume of the whole thyroid gland was calculated. Then its sonomorphology was assessed in B-mode regarding the following aspects: 
homogeneity or inhomogeneity, complex echogenicity and possible suspect nodules. If a lesion seemed to be solid, its size was measured in three dimensions. The critical diameter is determined to be larger than $10 \mathrm{~mm}$. The morphology of the lesion in B-mode was assessed using the TIRADS criteria $[3,21]$ : structure of lesion-margins, echogenicity, micro-calcifications and possible complex cystic sections. The marginal vascularization was evaluated and compared to the central sections of the lesion. Therefore, the flow parameters were set as low as possible (low PRF scale, GEN adapted, low filters) in order to avoid blooming artefacts. Compared to the surrounding thyroid tissue the blood flow in the lesion was rated as hypo-, iso- or hypervascularization. Afterwards stiff regions of the nodule were examined using strain and shear-wave elastography.

\subsection{CEUS examination technique}

For CEUS 1-2.4 ml sulfurhexafluorid microbubbles (SonoVue®, Bracco, Milan, Italy) along with $10 \mathrm{ml}$ sodium chloride were injected into a cubital vein. As long as there was only one suspicious lesion, several Cine-loops were recorded during the CEUS examination: the first one during the early arterial phase 10 to 15 seconds after the bolus injection, the second one during the late arterial phase 45 seconds after the injection and the last during the venous phase up to one minute after the bolus injection. In cases with more than one lesion, the replenishment was captured using FLASH kinetics. In order to detect a wash-out, late recordings were created up to 5 minutes after the bolus injection with intermittent Cine-loops lasting 10 seconds. Wash-out is defined as a lower contrast enhancement compared to the surrounding thyroid tissue. A malignant lesion had to have the following characteristics: irregular vascularization, missing or diffuse margins, wash-out starting in the late arterial phase, increasing until the venous phase $[3,8-10,18,21]$.

The remaining contrast 3 to 5 minutes after the bolus injection was utilized to capture the tumorvascularization on high-resolution Power Doppler, HR-flow and Glazing flow.

\subsection{Statistical analysis}

The data was statistically analyzed with Microsoft Excel 2016 (Microsoft Corporation, Redmond, WA). Descriptive analysis was used for further characterization of lesion parameters. Arithmetic means, standard deviation and $95 \%$ confidence intervals were calculated. Paired (data from the same group of patients) and unpaired (data from different groups) two-sided $t$-tests were performed for statistical comparisons. Probabilities less than 0.05 were considered significant.

\section{Results}

33 patients (13 males, 20 females; age 29- 77 years; mean 55 years) were included in this study. Interor postoperative histopathological analysis proved 37 thyroid nodules among them. 28 of them were benign nodules $(56.1 \pm 12.5$ years), 3 were adenomas $(58.7 \pm 4.0$ years) and 4 were diagnosed with carcinomas (47.0 \pm 15.0 years). Three carcinomas were histologically identified as papillary thyroid carcinomas and the last one as a medullary thyroid carcinoma. Every adenoma, carcinoma and 67.86\% of the patients with regressive nodules underwent a total or hemithyroidectomie. No peri- or postoperative complications were reported. The volume of the thyroid gland ranged between 6.6 to $401.3 \mathrm{~cm}^{2}$ (mean $72.6 \pm 92.0 \mathrm{~cm}^{2}$ ). The size, location and other B-mode findings like micro-calcifications of the thyroid lesions, echogenicity and vascularization of the lesions are summarized in Table 1.

The diameters of the benign regressive thyroid nodules ranged from 6 to $104 \mathrm{~mm}$, the adenoma diameter from 9 to $40 \mathrm{~mm}$ and the carcinoma diameter from 19 to $34 \mathrm{~mm}$. The average values for all lesions do not differ, and ranged from $22 \mathrm{~mm}$ for adenomas to $27.5 \mathrm{~mm}$ for carcinomas. 
Table 1

Demographical and ultrasound data for the three patient groups

\begin{tabular}{lccc}
\hline & Benign & Adenoma & Carcinoma \\
\hline $\mathrm{n}$ & 30 & 3 & 4 \\
Age [years] & $56.1 \pm 12.5$ & $58.7 \pm 4.0$ & $47.0 \pm 15.0$ \\
Lesion size [cm] & $2.75 \pm 2.13$ & $2.2 \pm 1.61$ & $2.58 \pm 0.62$ \\
Echogenicity margin & $1.89 \pm 0.46$ & $1.5 \pm 0.5$ & $1.38 \pm 0.48$ \\
Echogenicity center & $1.64 \pm 0.49$ & $1.5 \pm 0.5$ & $1.34 \pm 0.25$ \\
Vascularization margin & $1.94 \pm 0.45$ & $2.5 \pm 0.87$ & $2.88 \pm 0.25$ \\
Vascularization center & $1.31 \pm 0.62$ & $2.0 \pm 0.87$ & $1.63 \pm 0.75$ \\
Micro-calcifications & $7.14 \%$ & $33.3 \%$ & $100 \%$ \\
Complete or partial wash-out & $25 \%$ & $33.3 \%$ & $75 \%$ \\
\hline
\end{tabular}

The age of the patients did not differ significantly.

For rating of echogenicity, a scale from 1 to 3 was defined. In this scale 1 is used for a complete lack of echogenicity (echofree), 1.5 for lower echogenicity (hypoechoic), 2 for the same echogenicity as the surrounding thyroid tissue (isoechoic), 2.5 and 3 for higher echogenicity (hyperechoic). The echogenicity values of benign regressive nodules, adenomas and carcinomas were compared marginally and centrally. Only the benign regressive nodules reached values higher than 2 . The average echogenicity of thyroid carcinomas, especially marginally, was lower than the adenoma echogenicity values, which were lower than the benign regressive thyroid nodule values. Only the values at the rim of the carcinoma lesions were significantly different from the benign nodules $(p<0.0058)$.

For rating of the vascularization, a scale from 1 to 3 was defined. In this scale 1 is used for a complete lack of vascularization, 1.5 for lower vascularization (hypovascularization), 2 for the same vascularization as the surrounding thyroid tissue, 2.5 for higher vascularization (hypervascularization) and 3 for an extreme hypervascularization. The vascularization values of regressive thyroid nodules, adenomas and carcinomas were compared at the margin and centrally. The diagram shows a higher marginal vascularization for the thyroid carcinomas, which ranges from 2.5 to 3 . For benign regressive thyroid nodules and adenomas, the vascularization values started low from 1 and 1.5. The examined adenomas showed the highest average central vascularization. Only the values at the rim of the carcinoma lesions were significantly different from the benign nodules $(p<0.00036)$. Microcalcifications were visible in every examined carcinoma but only in one third of the included adenomas and even in a lower percentage of benign thyroid lesions. A complete or partial wash-out phenomenon occurred in 3 out of 4 carcinomas, in 1 out of 3 adenomas and in 7 out of 28 benign regressive thyroid nodules.

In addition, strain and shear-wave elastography were performed. The values are evaluated in Table 2.

Carcinomas tended to be stiffer (in $\mathrm{kPa}$ ) than the regressive benign nodules $(p=0.085)$; no differences were found between adenoma and carcinoma or benign nodules.

The thyroid carcinomas reached the highest elastography values in $\mathrm{kPa}$ in relation to the surrounding thyroid tissue regarding the center $(p=0.0000183)$ and margin $(p=0.0000048)$ of the lesion. Similar results were found for the elastography values in $\mathrm{m} / \mathrm{s}$.

Values of shear-wave elastography measurement from thyroid nodules in comparison to the surrounding tissue are presented in Fig. 1.

The thyroid carcinomas reached the highest absolute elastography values in $\mathrm{kPa}$ and $\mathrm{m} / \mathrm{s}$. Comparing the central and marginal $\mathrm{kPa}$ values to the surrounding thyroid tissue, the adenomas show values almost twice as high and the carcinomas more than twice as high as the benign regressive thyroid nodules. 
Table 2

Elastography data for the three patient groups

\begin{tabular}{lccc}
\hline & Benign & Adenoma & Carcinoma \\
\hline $\mathrm{n} \quad 30$ & 3 & 4 \\
$\quad$ Elastography [m/s] & & & \\
$\quad$ Center & $3.61 \pm 1.29$ & $3.93 \pm 1.03$ & $4.52 \pm 0.63$ \\
$\quad$ Margin & $3.51 \pm 2.31$ & $3.78 \pm 0.65$ & $4.34 \pm 0.81$ \\
$\quad$ Surrounding & $2.31 \pm 1.09$ & $1.79 \pm 0.57$ & $1.93 \pm 0.67$ \\
Elastography [kPa] & & & \\
$\quad$ Center & $44.5 \pm 30.8$ & $48.2 \pm 20.4$ & $64.3 \pm 22.3$ \\
$\quad$ Margin & $40.1 \pm 21.9$ & $40.9 \pm 13.5$ & $60.1 \pm 21.8$ \\
$\quad$ Surrounding & $18.4 \pm 20.3$ & $11.2 \pm 8.5$ & $12.3 \pm 6.5$ \\
\hline
\end{tabular}

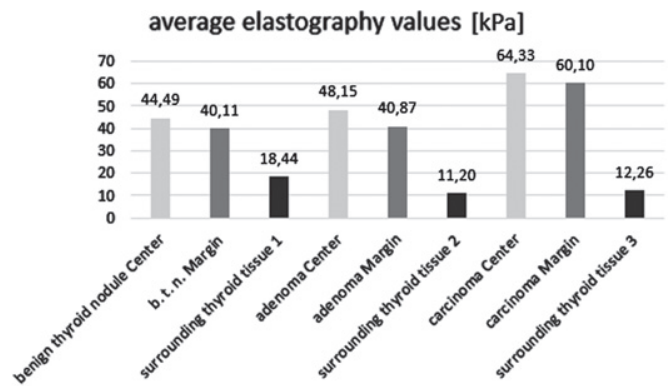

average elastography values $[\mathrm{kPa}]$ compared to the surrounding tissue

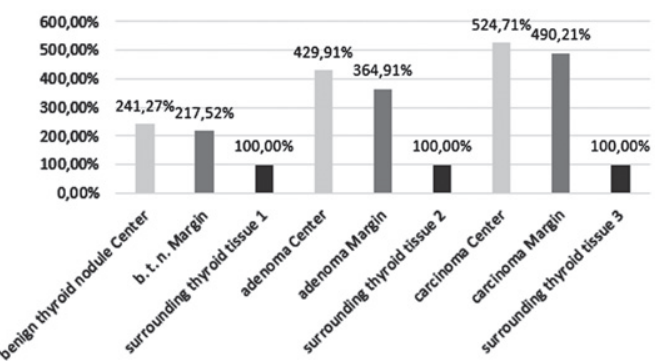

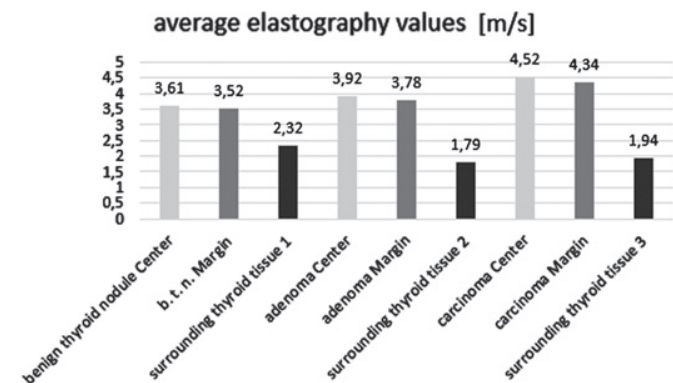

average elastography values $[\mathrm{m} / \mathrm{s}]$ compared to the surrounding tissue

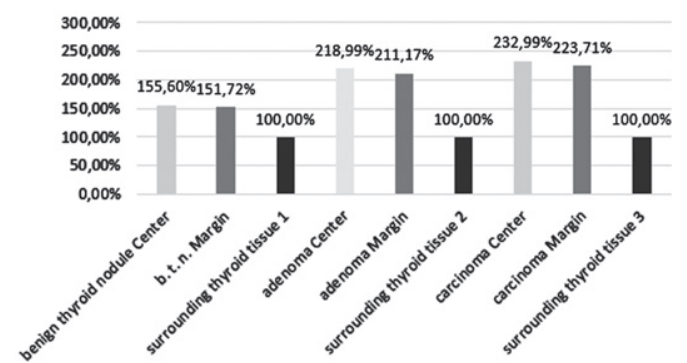

Fig. 1. Average absolute and relative shear-wave elastography values of the different thyroid lesions.

\subsection{Adenoma}

The adenoma diameters ranged from 9 to $40 \mathrm{~mm}$ (mean $22 \pm 16 \mathrm{~mm}$ ). They were equally located in the right and left thyroid lobe. Micro-calcifications were found in only one adenoma. Two of three adenomas showed an incomplete or diffuse margin and one adenoma had an irregular morphological structure (Fig. 2). The adenomas did not show consistent echogenicity: One was completely echofree, one was hypoechoic (Fig. 2) and one isoechoic.

Regular marginal as well as irregular central hypervascularization was detected in two adenomas after applying CCDS (Fig. 3 a-b). The last adenoma was hypovascularized.

One adenoma was extremely hardened centrally and two were moderately hardened centrally in strain elastography. One adenoma was slightly hardened marginally and two were moderately hardened marginally (Fig. 4 a). 


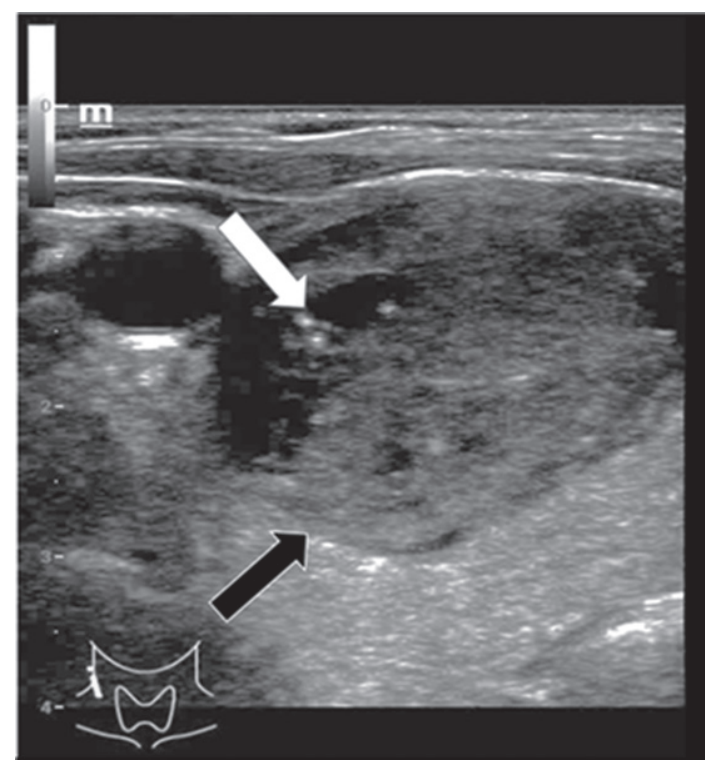

Fig. 2. B-mode examination of a right thyroid lobe in cross section of a 61-year-old patient with histo-pathologically proven thyroid adenoma. Micro-calcifications (white arrow) as well as a rather incomplete margin (black arrow) can be spotted in this image. There are strong differences between the echogenicity of the adenoma, in particular its margins, and the surrounding thyroid tissue.

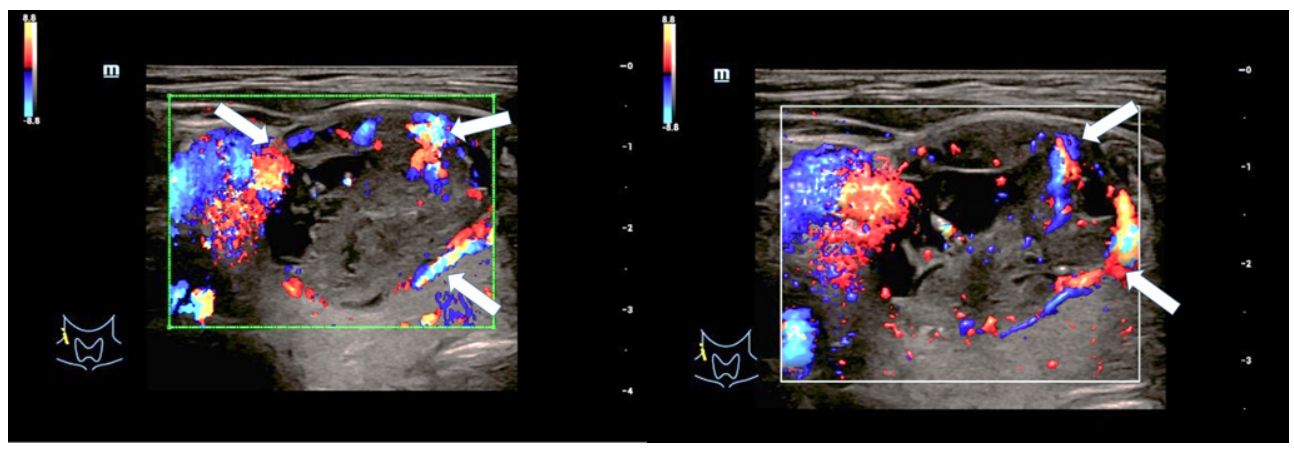

a

b

Fig. 3. CCDS (left/a) and Glazing-Flow (right/b) show an irregular hypervascularization (white arrows), especially occuring at the margins of a 61-year-old patient with a histopathologically proven thyroid adenoma.

Performing shear-wave elastography the adenomas showed lower values than the carcinomas (Fig. $5 \mathrm{a}-\mathrm{d}$ ): The tissue velocity of the adenomas ranged from $2.86 \mathrm{~m} / \mathrm{s}$ to $3.85 \mathrm{~m} / \mathrm{s}$ (mean $3.32 \pm 0.5 \mathrm{~m} / \mathrm{s}$ )

In CEUS two adenomas had no wash-out and the other one showed a partial wash-out phenomenon during the late venous phase (Fig. 6 a-b).

\subsection{Carcinoma}

The carcinoma diameters ranged from 19 to $33 \mathrm{~mm}$ (mean $26 \pm 6 \mathrm{~mm}$ ). The carcinomas were located in the right thyroid lobe. In B-mode micro-calcifications were found in every carcinoma. All of the carcinomas showed either diffuse or no margins. Half of the carcinomas had an irregular sono-morphological structure and the echogenicity of the carcinomas was equally characterized as hypoechoic or echofree (Fig. 7 a-b). 


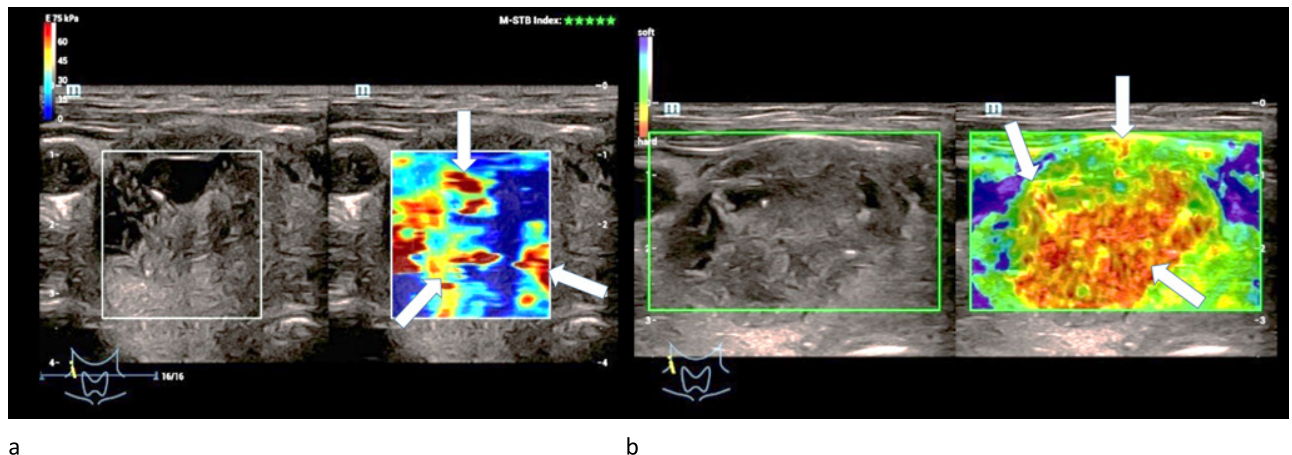

Fig. 4. Strain- (left/a) and shear-wave elastography (right/b) on a right thyroid lobe from a 61-year-old patient with histopathologically proven thyroid adenoma. Irregular hard areas (white arrows) can be found in both modi. In strain the hardenings are predominently located along the right margin of the lesion whereas in shear-wave the whole lesion seems to be hardened.
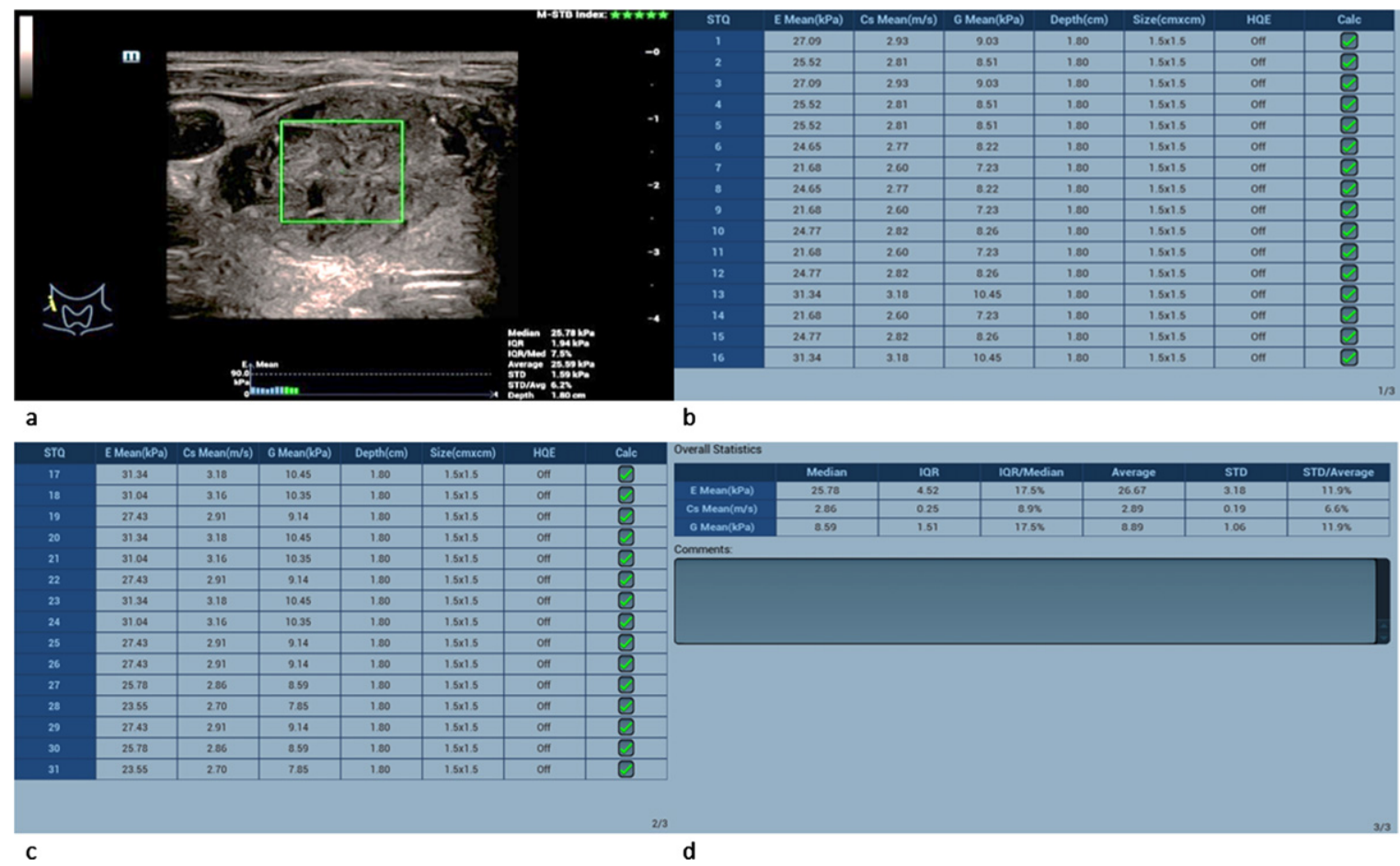

Fig. 5. 61-year-old patient with histopathologically proven thyroid adenoma. Regions of interest (ROIs) are set across the lesion (a). 31 different measurements of the tissue stiffness were performed ( $b$ and c). The average measured shear-wave elastography values (d) are $25.78 \mathrm{kPa}$ and $2.86 \mathrm{~m} / \mathrm{s}$. These values slightly surpace the malignancy limit of $2.5 \mathrm{~m} / \mathrm{s}$ but lie below the malgnancy limit of $30 \mathrm{kPa}$.

The four carcinomas showed an irregular extreme hypervascularization along their margins as well as an irregular central normo- or hypervascularization in CCDS (Fig. 8 a-b). Furthermore, the capillarization of all carcinomas was very irregular.

Half of the carcinomas were moderately hardened and the other half was extremely hardened centrally and marginally in strain elastography (Fig. 9 a).

The measured shear-wave elastography values of the carcinomas significantly surpassed the adenomas' values as well as the malignancy limit of $2.5 \mathrm{~m} / \mathrm{s}$ and $30 \mathrm{kPa}$ (Fig. $10 \mathrm{a}-\mathrm{d}$ ): Two carcinomas had values between 3 and $4 \mathrm{~m} / \mathrm{s}$ and the other two between 4 and $5 \mathrm{~m} / \mathrm{s}$. 


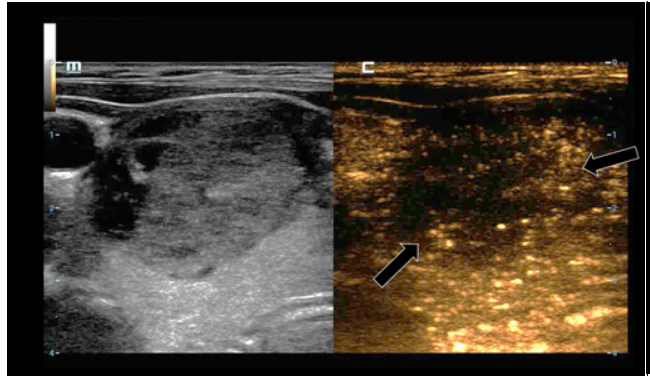

a

T1: 0:10

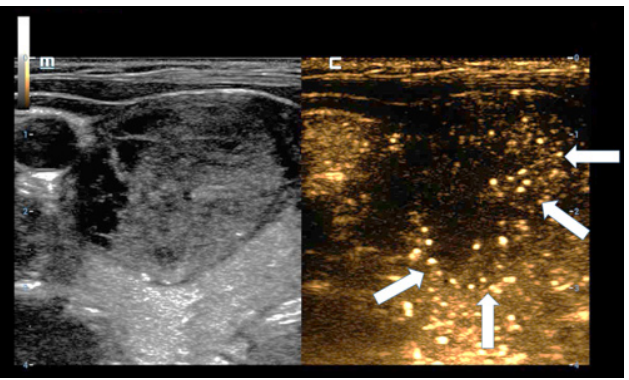

T1: 0:14

Fig. 6. 61-year-old patient with histopathologically proven thyroid adenoma. In the first picture (left/a) the CEUS kinetics of the lesion are depicted only seconds after the bolus injection of $1.5 \mathrm{ml}$ SonoVue®. The margins of the lesion did not absorb a lot of microbubbles (black arrows). In the subsequent picture (right/b) a contrast enhancement of the lesions' margins called wash-in (white arrows) occurs. A wash-out phenomenon did not take place.

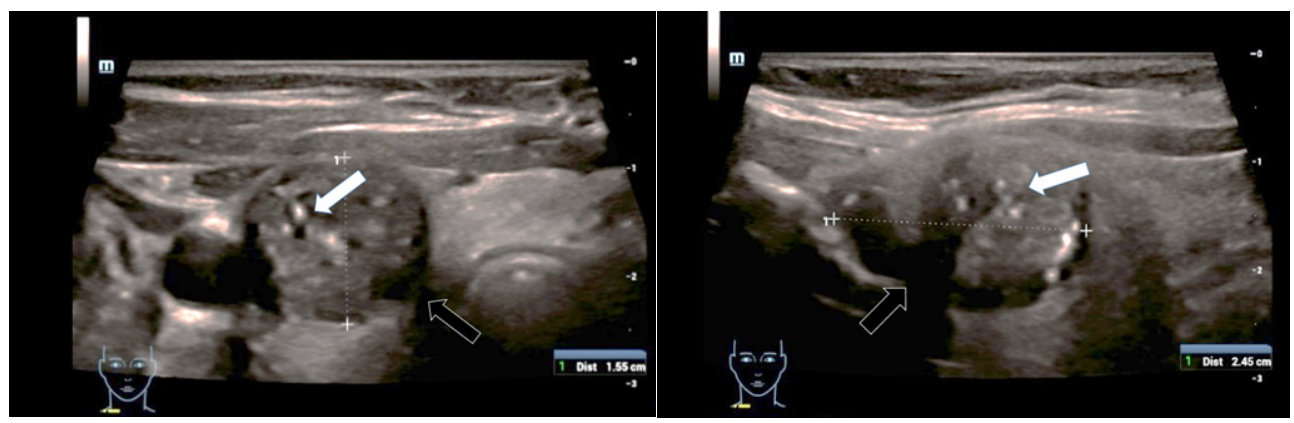

b

Fig. 7. B-mode examination of a right thyroid lobe in cross section (left/a) and longitudinal view (right/b) of a 69-year-old patient with histo-pathologically proven papillary thyroid cancer. Micro-calcifications (white arrows), a diffuse, incomplete margin (black arrows) and a marginal notably reduced echogenicity compared to the surrounding thyroid tissue can be found. These are B-mode signs for malignancy.

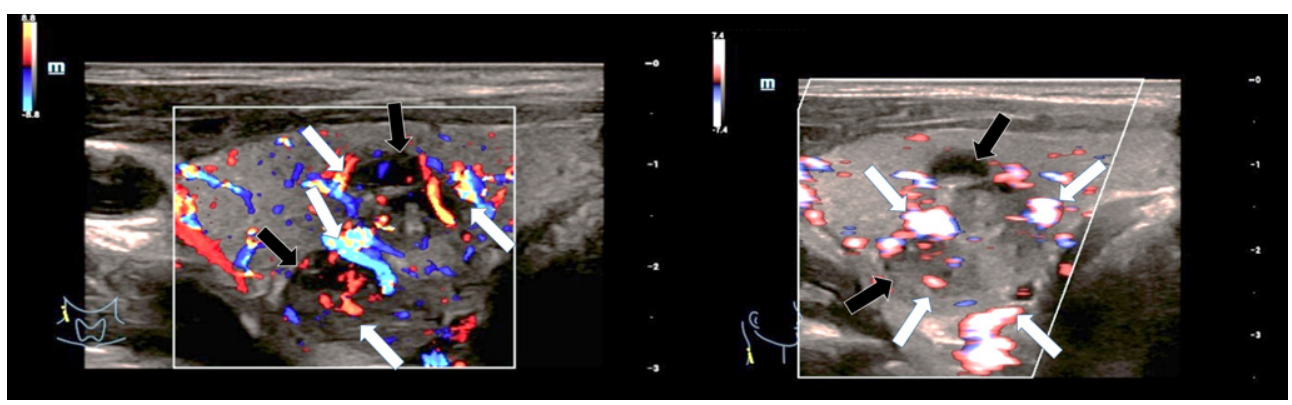

b

Fig. 8. 29-year-old patient with histo-pathologically proven bifocal (black arrows) medullary thyroid cancer. CCDS (left/a) and HR-Flow (right/b) showed an irregular hypervascularization (white arrows), especially occuring at the margins. This also points at malignancy. 


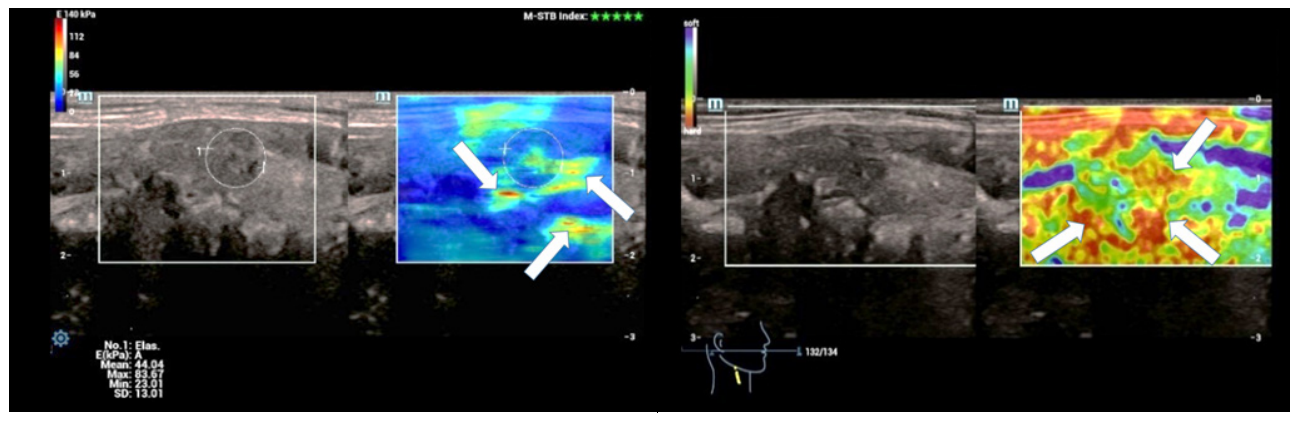

a

b

Fig. 9. 36-year-old patient with histo-pathologically proven papillary thyroid cancer. Strain- (left/a) and shear-wave elastography (right/b) of a right thyroid lobe showed irregular hard areas (white arrows) in both modi. They are predominently located at the margins.
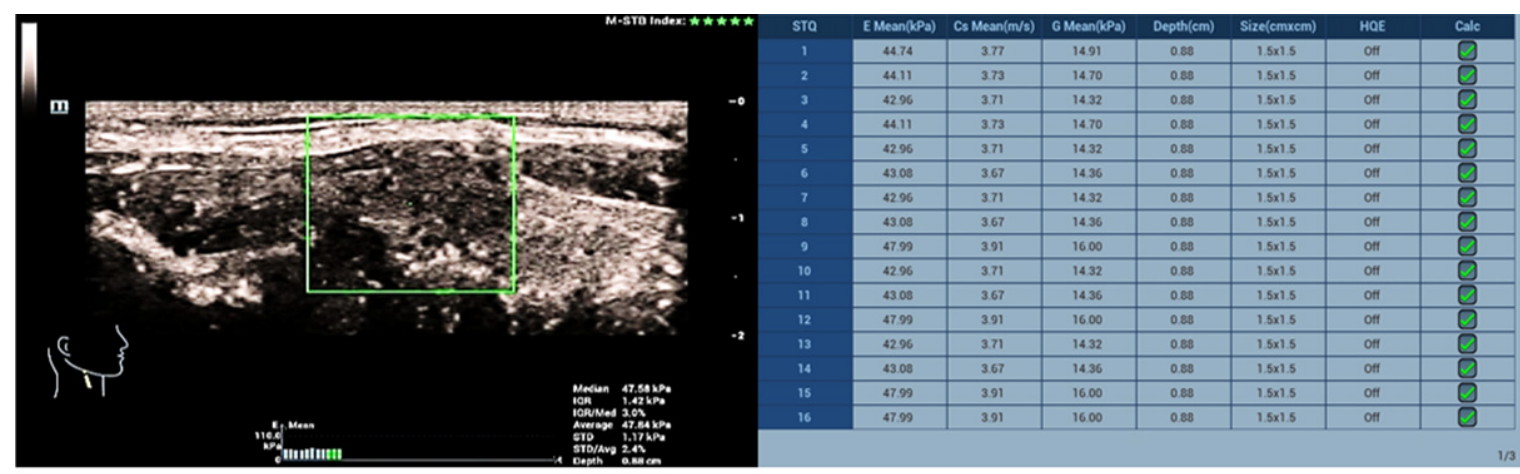

a

b

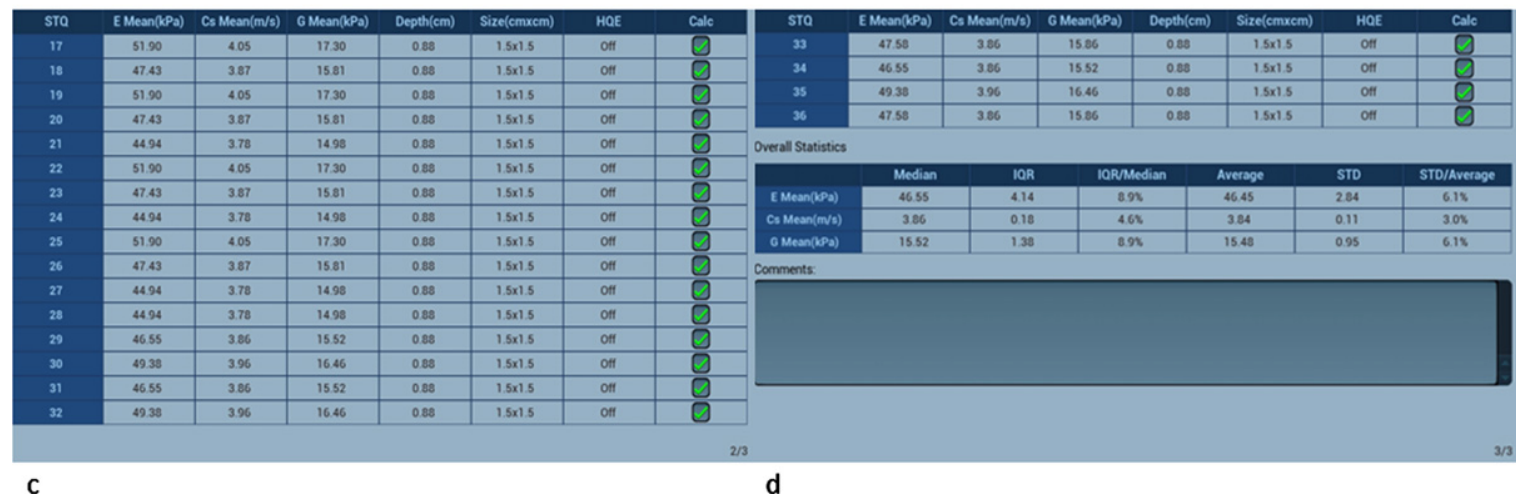

Fig. 10. 36-year-old patient with papillary thyroid cancer (same patient as in Fig. 3). Regions of interest (ROIs) are set across the lesion (a). 36 different measurements of the tissue stiffness were performed ( $b, c$ and d). The average measured shear-wave elastography values (d) clearly surpass the malignancy limit of $2.5 \mathrm{~m} / \mathrm{s}$ and $30 \mathrm{kPa}$.

In CEUS two carcinomas showed a complete wash-out in the late arterial phase (Fig. 11 a-b), whereas the other two showed only a partial or no wash-out phenomenon.

\section{Discussion}

The frequency of thyroid carcinoma varies with a risk in between 1 and $10 \%$ depending on multiple factors like familial predisposition, multimorbidity, and condition after radio-chemotherapy or 


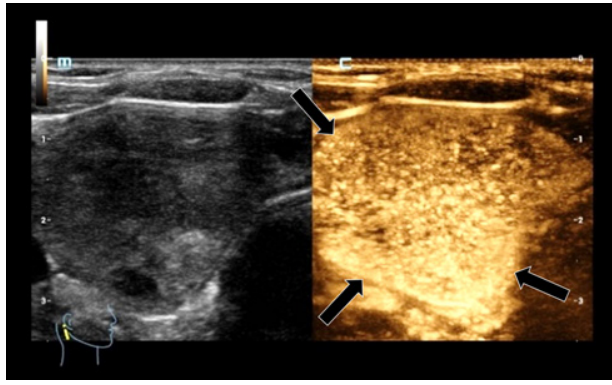

a

T2: $0: 23$

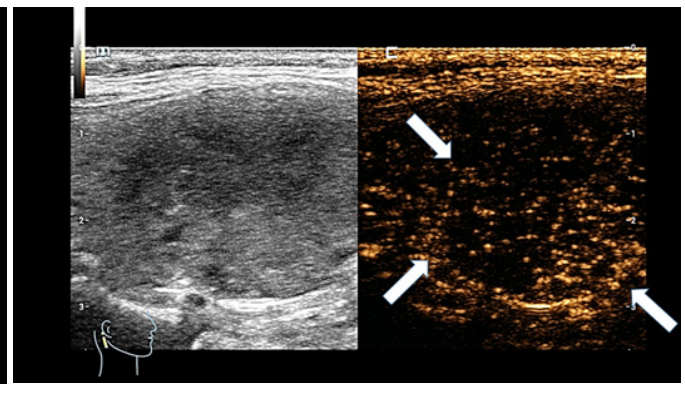

T2: $1: 40$

Fig. 11. 44-year-old patient with histo-pathologically proven papillary thyroid cancer. After the bolus injection of $1.5 \mathrm{ml}$ SonoVue ${ }^{\circledR}$ the CEUS-kinetics of the lesion were documented: an early irregular marginal and central enhancement phenomenon (black arrows) called wash-in (left/a) took place. An early wash-out (white arrows) during the late arterial phase (right/b) was visible.

treatment of a lymphoma. 4 of the 33 included patients of this study were diagnosed with thyroid cancer, showing a frequency of $12.12 \%$ in the examined group. The reason for this high prevalence of thyroid carcinomas in our study are the preliminary diagnostics as well as a rising number of thyroid carcinomas worldwide. In the USA the incidence for developing a thyroid carcinoma increased from 4.56 per 100000 persons during the years 1974-1977 to 14.42 per 100000 persons during the years 2010-2013. That is an average rise of 3.6\% per year from 1974 to 2013 [4]. Spotting thyroid carcinomas can be difficult as usually no early clinical symptoms occur. Nevertheless, using the modern ultrasound diagnostics according to the patients' risk, a sufficiently high number of tumor suspect lesions was detected. The modern sonographic diagnostics include B-mode, CCDS, high-resolution ultrasound techniques like HR-flow and Glazing flow, CEUS and elastography.

Regarding the TIRADS-criteria every lesion with a size larger than $10 \mathrm{~mm}$, including intracystic papilloma cases, showed sonomorphological suspect findings hinting at solid tumors [3, 21].

According to recent studies about elastography, performed on the thyroid gland, the limit stiffness value of a malignant lesion is $2.5 \mathrm{~m} / \mathrm{s}$ or $30 \mathrm{kPa}$. The diagnostic confidence for this varies depending on the study. The average value is $85 \%$ [5-7, 23, 24]. Regardless we also found adenomas and benign lesions in our patient group with augmented values in tissue speed and stiffness while using shear-wave elastography.

Several research groups published their results about the differentiation between benign and malignant thyroid lesions. B-mode findings like a hypoechoic margin and micro-calcifications or an irregular hypervascularization of the lesion in CCDS were described as possible carcinoma characteristics [3, 16-18, 21]. In CEUS a wash-out phenomenon during the late arterial or venous phase strongly indicates malignancy according to multiple studies [8-10]. However, using B-mode, elastography, CCDS and CEUS did not lead up to a final evaluation of a lesion as benign or malignant. Further multimodal sonographical studies are required to determinate certain contrast conduction and hemodynamic patterns as well as better elastography cut-off values for an unambiguous differentiation between benignity and malignancy. Nevertheless, the diagnostic accuracy of all four sonographic imaging modes, especially CEUS, for evaluating a thyroid lesion has significantly improved [11].

For using CEUS in the evaluation of vascularization we first had to differentiate between microand macrovascularization. In order to decide whether the nodule was benign or malignant the conduct patterns of the lesion after bolus injection of the contrast agent was observed from the early arterial phase to the late venous phase. An irregular early hypervascularization alongside a wash-out, which started in the late arterial or early venous phase and continued to the late venous phase, was an important factor for malignancy. The average sensitivity of these criteria varies between 60 and $95 \%$ with an average value 
of $85 \%$ [12-15]. However, in preliminary examinations even some patients with necrotic or complex cystic adenomas showed a partial wash-out. The standard criteria for the evaluation of a thyroid lesion as carcinoma are irregular hypervascularization patterns, an expansion into the depth, low echo, solid margins and most importantly an irregular wash-out of the hypervascularized tumor margins [16-18]. Furthermore, regarding the TIRADS criteria an inhomogeneous structure and micro-calcifications are also factors for malignancy $[3,21]$. Regarding our study several malignancy criteria can be confirmed as every examined malignant carcinoma showed micro-calcifications and a hypoechoic or echofree margin in B-mode as well as an irregular extreme hypervascularization along its margins in CCDS. The wash-out phenomenon as a malignancy criteria was mainly confirmed as well because a complete or partial wash-out phenomenon was observed in 3 of 4 examined carcinomas.

In CEUS adenomas usually show a marginal enhancement during the arterial phase as well, but with no disappearance of that enhancement during the late arterial or venous phase [20]. No visible or only a partial wash-out are typical for thyroid adenomas. One of the examined adenomas showed a partial marginal wash-out, whereas the other two had no visible wash-out phenomenon.

The findings were histologically examined with high diagnostic confidence after they were surgically removed. However, a frozen section analysis was always performed during the operation for deciding whether only one lobe or the whole thyroid gland had to be removed. In most studies histological or cytological diagnostics were performed using fine needle aspiration (FNA) or sonographic biopsy. Nevertheless, the technique used in our study is considered equally.

The retrospective approach and the time consuming, extensive, on high-end-technology depending sonographic examination can be seen as limitations for this study. Furthermore, the examinations had to be performed by an experienced sonographer on a preliminary examined group of patients.

In conclusion, the modern, extensive, multimodal ultrasound diagnostics of thyroid lesions should be available for future clinical applications and provides an opportunity for interesting further multicenter studies.

\section{Conflict of interest}

The authors have no conflict of interest to report.

\section{References}

[1] Brander AE, Viikinkoski VP, Nickels JI, Kivisaari LM. Importance of thyroid abnormalities detected at US screening: a 5-year follow-up. Radiology. 2000;215(3):801-6.

[2] Hornung M, Jung EM, Georgieva M, Schlitt HJ, Stroszczynski C, Agha A. Detection of microvascularization of thyroid carcinomas using linear high resolution contrast-enhanced ultrasonography (CEUS). Clin Hemorheol Microcirc. 2012;52(2-4):197-203.

[3] Russ G, Bonnema SJ, Erdogan MF, Durante C, Ngu R, Leenhardt L. European Thyroid Association Guidelines for Ultrasound Malignancy Risk Stratification of Thyroid Nodules in Adults: The EU-TIRADS. Eur Thyroid J. 2017;6(5):225-37.

[4] Lim H, Devesa SS, Sosa JA, Check D, Kitahara CM. Trends in Thyroid Cancer Incidence and Mortality in the United States. 1974-2013. JAMA. 2017;317(13):1338-48.

[5] Zhan J, Jin JM, Diao XH, Chen Y. Acoustic radiation force impulse imaging (ARFI) for differentiation of benign and malignant thyroid nodules-A meta-analysis. Eur J Radiol. 2015;84(11):2181-6.

[6] Dong FJ, Li M, Jiao Y, Xu JF, Xiong Y, Zhang L, Luo H, Ding ZM. Acoustic Radiation Force Impulse imaging for detecting thyroid nodules: a systematic review and pooled meta-analysis. Med Ultrason. 2015;17(2):192-9.

[7] Bojunga J, Herrmann E, Meyer G, Weber S, Zeuzem S, Friedrich-Rust M. Real-time elastography for the differentiation of benign and malignant thyroid nodules: a meta-analysis. Thyroid. 2010;20(10):1145-50.

[8] Wiesinger I, Kroiss E, Zausig N, Hornung M, Zeman F, Stroszczynski C, Jung EM. Analysis of arterial dynamic microvascularization with contrast-enhanced ultrasound (CEUS) in thyroid lesions using external perfusion software: First results. Clin Hemorheol Microcirc. 2016;64(4):747-55. 
[9] Wendl CM, Janke M, Jung W, Stroszczysnski C, Jung EM. Contrast-enhanced ultrasound with perfusion analysis for the identification of malignant and benign tumours of the thyroid gland. Clin Hemorheol Microcirc. 2015;63(2):113-21.

[10] Nemec U, Nemec SF, Novotny C, Weber M, Czerny C, Krestan CR. Quantitative evaluation of contrast-enhanced ultrasound after intravenous administration of a microbubble contrast agent for differentiation of benign and malignant thyroid nodules: assessment of diagnostic accuracy. Eur Radiol. 2012;22(6):1357-65.

[11] Schleder S, Janke M, Agha A, Schacherer D, Hornung M, Schlitt HJ, Stroszczynski C, Schreyer AG, Jung EM. Preoperative differentiation of thyroid adenomas and thyroid carcinomas using high resolution contrast-enhanced ultrasound (CEUS). Clin Hemorheol Microcirc. 2015;61(1):13-22.

[12] Pang T, Huang L, Deng Y, Wang T, Chen S, Gong X, Liu W. Logistic regression analysis of conventional ultrasonography, strain elastosonography, and contrast-enhanced ultrasound characteristics for the differentiation of benign and malignant thyroid nodules. PLoS One. 2017;12(12):e0188987

[13] Trimboli P, Castellana M, Virili C, Havre RF, Bini F, Marinozzi F, D’ Ambrosio F, Giorgino F, Giovanella L, Prosch H, Grani G, Radzina M, Cantisani V. Performance of contrast-enhanced ultrasound (CEUS) in assessing thyroid nodules: a systematic review and meta-analysis using histological standard of reference. Radiol Med. 2020;125(4):406-15.

[14] Xi X, Gao L, Wu Q, Fang S, Xu J, Liu R, Yang X, Zhu S, Zhao R, Lai X, Zhang X, Zhang B, Jiang Y. Differentiation of Thyroid Nodules Difficult to Diagnose With Contrast-Enhanced Ultrasonography and Real-Time Elastography. Front Oncol. 2020;10:112.

[15] Zhao RN, Zhang B, Jiang YX, Yang X, Lai XJ, Zhu SL, Zhang XY. Ultrasonographic Multimodality Diagnostic Model of Thyroid Nodules. Ultrason Imaging. 2019;41(2):63-77.

[16] Ginat DT, Butani D, Giampoli EJ, Patel N, Dogra V. Pearls and pitfalls of thyroid nodule sonography and fine-needle aspiration. Ultrasound Q. 2010;26(3):171-8.

[17] Smith-Bindman R, Lebda P, Feldstein VA, Sellami D, Goldstein RB, Brasic N, Jin C, Kornak J. Risk of thyroid cancer based on thyroid ultrasound imaging characteristics: results of a population-based study. JAMA Intern Med. 2013;173(19):1788-96.

[18] Tay SY, Chen CY, Chan WP. Sonographic criteria predictive of benign thyroid nodules useful in avoiding unnecessary ultrasound-guided fine needle aspiration. J Formos Med Assoc. 2015;114(7):590-7.

[19] Wu Q, Li Y, Wang Y. Diagnostic value of "absent" pattern in contrast-enhanced ultrasound for the differentiation of thyroid nodules. Clin Hemorheol Microcirc. 2016;63(4):325-34.

[20] Zhang B, Jiang YX, Liu JB, Yang M, Dai Q, Zhu QL, Gao P. Utility of contrast-enhanced ultrasound for evaluation of thyroid nodules. Thyroid. 2010;20(1):51-7.

[21] Zhao H, Liu X, Lei B, Cheng P, Li J, Wu Y, Ma Z, Wei F, Su H. Diagnostic performance of thyroid imaging reporting and data system (TI-RADS) alone and in combination with contrast-enhanced ultrasonography for the characterization of thyroid nodules. Clin Hemorheol Microcirc. 2019;72(1):95-106.

[22] Shi YZ, Jin Y, Zheng L. Partially cystic thyroid nodules on ultrasound: The associated factors for malignancy. Clin Hemorheol Microcirc. 2020;74(4):373-81.

[23] Zhang WB, Li JJ, Chen XY, He BL, Shen RH, Liu H, Chen J, He XF. SWE combined with ACR TI-RADS categories for malignancy risk stratification of thyroid nodules with indeterminate FNA cytology. Clin Hemorheol Microcirc. 2020;76(3):381-90.

[24] Lian KM, Lin T. Value of image-pro plus for assisting virtual touch tissue imaging in the diagnosis of thyroid nodules. Clin Hemorheol Microcirc. 2021;77(2):143-51. 